\title{
Some Results on Vertex Equitable Labeling
}

\author{
P. Jeyanthi ${ }^{1}$, A. Maheswari ${ }^{2}$ \\ ${ }^{1}$ Research Centre, Department of Mathematics, Govindammal Aditanar College for Women, Tiruchendur, India \\ ${ }^{2}$ Department of Mathematics, Kamaraj College of Engineering and Technology, Virudhunagar, India \\ Email: jeyajeyanthi@rediffmail.com, bala_nithin@yahoo.co.in
}

Received December 15, 2011; revised January 23, 2012; accepted February 18, 2012

\section{ABSTRACT}

Let $G$ be a graph with $p$ vertices and $q$ edges and let $A=\left\{0,1,2, \cdots,\left\lceil\frac{q}{2}\right\rceil\right\}$. A vertex labeling $f: V(G) \rightarrow A$ is said to be a vertex equitable labeling of $G$ if it induces an edge labeling $f^{*}$ given by $f^{*}(u v)=f(u)+f(v)$ such that $\left|v_{f}(a)-v_{f}(b)\right| \leq 1$ and $f^{*}(E)=\{1,2,3, \cdots, q\}$, where $v_{f}(a)$ is the number of vertices $v$ with $f(v)=a$ for $a \in A$. A graph $G$ is said to be a vertex equitable graph if it admits vertex equitable labeling. In this paper, we establish the vertex equitable labeling of a $T_{p}$-tree, $T \odot \overline{K_{n}}$ where $T$ is a $T_{p}$-tree with even number of vertices, bistar $B(n, n+1)$, the caterpillar $S\left(x_{1}, x_{2}, \cdots, x_{n}\right)$ and crown $C_{n} \odot K_{1}, P_{n}^{2}$.

Keywords: Vertex Equitable Labeling; Vertex Equitable Graph

\section{Introduction}

All graphs considered here are simple, finite, connected and undirected. We follow the basic notations and terminologies of graph theory as in [1]. The symbols $V(G)$ and $E(G)$ denote the vertex set and the edge set of a graph $G$. Let $G(p, q)$ be a graph with $p=|V(G)|$ vertices and $q=|E(G)|$ edges. A labeling $f$ of a graph $G$ is a mapping that assigns elements of a graph to the set of numbers (usually to positive or non-negative integers). If the domain of the mapping is the set of vertices (the set of edges) then we call the labeling vertex labeling (edge labeling). The labels of the vertices induce labels of the edges. There are several types of labeling. A detailed survey of graph labeling can be found in [2]. A vertex labeling $f$ is said to be difference labeling if it induces the label $|f(x)-f(y)|$ for each edge $x y$ which is called as weight of the edge $x y$.

A difference labeling $f$ of a graph $G$ is said to be $k$-equitable if for each weight induced by $f$ on the edges of $G$ appears exactly $k$ times. If a graph $G$ has a $k$-equitable labeling then $G$ is said to be $k$-equitable. Equitable labeling of graphs was introduced by Bloom and Ruiz in [3]. A brief summary of definitions which are useful for the present study is given below.

Definition 1.1 [4] Let $T$ be a tree and $u_{0}$ and $v_{0}$ be two adjacent vertices in $T$. Let $u$ and $v$ be two pendant vertices of $T$ such that the length of the path $u_{0}-u$ is equal to the length of the path $v_{0}-v$. If the edge $u_{0} v_{0}$ is deleted from $T$ and $u$ and $v$ are joined by an edge $u v$, then such a transformation of $T$ is called an elementary parallel transformation (or an ept, for short) and the edge $u_{0} v_{0}$ is called transformable edge.

If by a sequence of ept's, $T$ can be reduced to a path, then $T$ is called a $T_{p}$ tree (transformed tree) and such sequence is regarded as a composition of mappings (ept's) denoted by $P$, is called a parallel transformation of $T$. The path, the image of $T$ under $P$ is denoted as $P(T)$.

A $T_{p}$ tree and a sequence of two ept's reducing it to a path are illustrated in Figure 1.

Definition 1.2 The corona $G_{1} \odot G_{2}$ of the graphs $G_{1}$ and $G_{2}$ is obtained by taking one copy of $G_{1}$ (with $p$ vertices) and $p$ copies of $G_{2}$ and then joining the $i^{\text {th }}$ vertex of $G_{1}$ to every vertex of the $i^{\text {th }}$ copy of $G_{2}$.

Definition 1.3 Caterpillar is a tree with the property that the removal of its pendant vertices leaves a path.

Definition 1.4 The square graph $G^{2}$ of a graph $G$ has the vertex set $V\left(G^{2}\right)=V(G)$ with $u, v$ adjacent in $G^{2}$ whenever $d(u, v) \leq 2$ in $G$.

$\lceil x\rceil$ denotes the smallest integer greater than or equal to $x$.

The concept of mean labeling was introduced by S. Somasundaram and R. Ponraj in [5] and further studied in [6-8]. A. Lourdusamy and M. Seenivasan introduced a vertex equitable labeling in [9]. In a vertex equitable labeling we use the labels $0,1,2, \cdots,\lceil q / 2\rceil$ for the vertices, 
a) A $T_{p}$-tree $T$

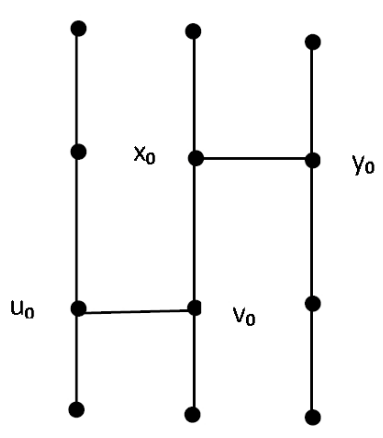

b) An ept $P_{1}(\mathrm{~T})$

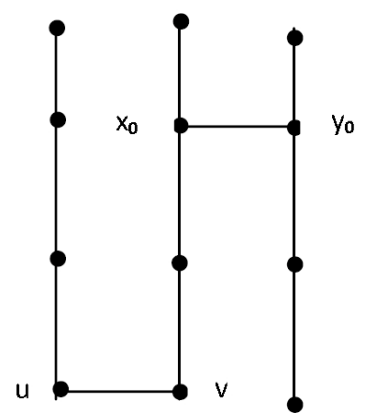

c) Second ept $P_{2}(\mathrm{~T})$

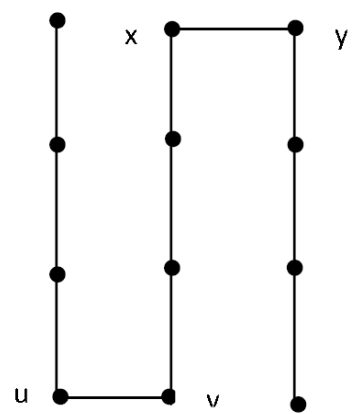

Figure 1. A $T_{p}$-tree and a sequence of two ept's reducing it to a path.

the number of times the different vertex labels appear cannot differ by more than one. The induced edge labels are defined as the sum of the incident vertex labels. They proved that the graphs like path, bistar $B(n, n)$, combs $P_{n} \odot K_{1}$, bipartite complete $K_{2, n}$, friendship graph $C_{3}^{(t)}$ for $t \geq 2$, quadrilateral snake,

$$
K_{2}+m K_{1}, K_{1, n} \cup K_{1, n+k}
$$

if and only if $1 \leq k \leq 3$, ladder graph $L_{n}=P_{n} \times K_{2}$, arbitrary super division of a path and cycle $C_{n}$ with $n \equiv 0$ or $3(\bmod 4)$ are vertex equitable. Also they proved that the graph $K_{1, n}$ if $n \geq 4$, Eulerian graph with $n$ edges where $n \equiv 1$ or $2(\bmod 4)$, the wheel $W_{n}$, the complete graph $K_{n}$ if $n>3$ and triangular cactus with $q$ edges where $q \equiv 0$ or 6 or $9(\bmod 12)$ are not vertex equitable. Moreover they proved that if $G$ is a graph with $p$ vertices and $q$ edges, $q$ is even and $p<\lceil q / 2\rceil+2$ then $G$ is not vertex equitable.

Definition 1.5 [9] Suppose $G$ is a graph with $p$ vertices and $q$ edges. Let $A=\{0,1,2, \cdots,[q / 2\rceil\}$. A vertex labeling $f: V \rightarrow A$ induces an edge labeling $f^{*}$ defined by $f^{*}(u v)=f(u)+f(v)$ for all edges uv. For $a \in A$, let $v_{f}(a)$ be the number of vertices $v$ with $f(v)=a$. A graph $G$ is vertex equitable if there exists a vertex labeling $f$ such that for all $a$ and $b$ in $A$,

$$
\left|v_{f}(a)-v_{f}(b)\right| \leq 1
$$

and the induced edge labels are $1,2,3, \cdots, q$.

P. Jeyanthi and A. Maheswari proved in $[10,11]$ that tadpoles, $C_{m} \oplus C_{n}$, armed crowns, $\left[P_{m} ; C_{n}^{2}\right]$ and, $\left\langle P_{m} \hat{o} K_{1, n}\right\rangle$, the graphs obtained by duplicating an arbitrary vertex and an arbitrary edge of a cycle $C_{n}$, total graph of $P_{n}$, splitting graph of $P_{n}$ and fusion of two edges of a cycle $C_{n}$ are vertex equitable graphs. In this paper, we establish the vertex equitable labeling of a $T_{p}$-tree, $T \odot \overline{K_{n}}$ where $T$ is a $T_{p}$-tree with even number of vertices, the bistar $B(n, n+1)$, the caterpillar $S\left(x_{1}, x_{2}, \cdots, x_{n}\right)$ and the crown $C_{n} \odot K_{1}, P_{n}^{2}$.

\section{Main Results}

Theorem 2.1 Let $G_{1}\left(p_{1}, 2 n\right)$ and $G_{2}\left(p_{2}, q_{2}\right)$ be any two vertex equitable graphs with equitable labeling $f$ and $g$ respectively. Let $u$ and $v$ be the vertices of $G_{1}$ and $G_{2}$ respectively such that $f(u)=n$ and $g(v)=0$. Then the graph $\left(G_{1}\right)_{f} *\left(G_{2}\right)_{g}$ obtained from $G_{1}$ and $G_{2}$ by identifying the vertices $u$ and $v$ is a vertex equitable graph.

Proof. Clearly $\left(G_{1}\right)_{f} *\left(G_{2}\right)_{g}$ has $2 n+q_{2}$ edges and $p_{1}+p_{2}-1$ vertices. Let

$$
\begin{aligned}
& V\left(G_{1}\right)=\left\{u, u_{i}: 1 \leq i \leq p_{1}-1\right\}, \\
& V\left(G_{2}\right)=\left\{v, v_{i}: 1 \leq i \leq p_{2}-1\right\} .
\end{aligned}
$$

Define $h: V\left(\left(G_{1}\right)_{f} *\left(G_{2}\right)_{g}\right) \rightarrow\left\{0,1,2, \cdots,\left\lceil\frac{2 n+q_{2}}{2}\right\rceil\right\}$

by $h\left(u_{i}\right)=f\left(u_{i}\right)$ for $1 \leq i \leq p_{1}-1, h(v)=f(u)$ and $h\left(v_{i}\right)=g\left(u_{i}\right)+n$ for $1 \leq i \leq p_{2}-1$. Clearly,

$$
v_{h}(a)= \begin{cases}v_{f}(a) & \text { if } 0 \leq a \leq n \\ v_{g}(a) & \text { if } n+1 \leq a \leq\left\lceil\frac{q_{2}}{2}\right\rceil+n .\end{cases}
$$

Therefore, $\left|v_{h}(a)-v_{h}(b)\right| \leq 1$ and the labels of the edges of the copy of $G_{1}$ are $1,2, \cdots, 2 n$ and the labels of the edges of the copy of $G_{2}$ are

$2 n+1,2 n+2, \cdots, 2 n+q_{2}$. Hence, $\left(G_{1}\right)_{f} *\left(G_{2}\right)_{g}$ is a vertex equitable graph.

Theorem 2.2 Let $G_{1}\left(p_{1}, 2 n+1\right)$ and $G_{2}\left(p_{2}, q_{2}\right)$ be any two vertex equitable graphs with equitable labeling $f$ and $g$ respectively. Let $u$ and $v$ be the vertices of $G_{1}$ and $G_{2}$ respectively such that $f(u)=n+1$ and $g(v)=0$. Then the graph $G$ obtained by joining $u$ and $v$ by an edge is vertex equitable.

Proof. Clearly $G$ has $2 n+2+q_{2}$ edges and $p_{1}+p_{2}$ vertices. Let $V\left(G_{1}\right)=\left\{u, u_{i}: 1 \leq i \leq p_{1}-1\right\}, V\left(G_{2}\right)=\left\{v, v_{i}: 1 \leq i \leq p_{2}-1\right\}$.

Define $h: V(G) \rightarrow\left\{0,1,2, \cdots,\left\lceil\frac{2 n+2+q_{2}}{2}\right\rceil\right\}$ 
by $h(w)=f(w), \quad$ if $\quad w \in V\left(G_{1}\right), h(w)=g(w)+n+1$ if $w \in V\left(G_{2}\right)$. The labels of the edges of the copy of $G_{1}$ are $1,2, \cdots, 2 n+1$ and the labels of the edges of the copy of $G_{2}$ are $2 n+3,2 n+4, \cdots, 2 n+2+q_{2}$ and

$$
h^{*}(u v)=h(u)+h(v)=2 n+2 .
$$

Hence, $G$ is a vertex equitable graph.

Theorem 2.3 Every $T_{p}$-tree is a vertex equitable graph.

Proof. Let $T$ be a $T_{p}$-tree with $n$ vertices. By the definition of a transformed tree there exists a parallel transformation $P$ of $T$ such that for the path $P(T)$ we have 1) $V(P(T))=V(T)$,2) $E(P(T))=\left(E(T)-E_{d}\right) \cup E_{p}$ where $E_{d}$ is the set of edges deleted from $T$ and $E_{p}$ is the set of edges newly added through the sequence

$P=\left(P_{1}, P_{2}, \cdots, P_{k}\right)$ of the epts $P$ used to arrive the path $P(T)$. Clearly, $E_{d}$ and $E_{p}$ have the same number of edges.

Now denote the vertices of $P(T)$ successively as $v_{1}, v_{2}, \cdots, v_{n}$ starting from one pendant vertex of $P(T)$ right up to the other.

For $1 \leq i \leq n$, define the labeling $f$ as

$$
f\left(v_{i}\right)= \begin{cases}\frac{i-1}{2} & \text { if } i \text { is odd } \\ \frac{i}{2} & \text { if } i \text { is even }\end{cases}
$$

Then $f$ is a vertex equitable labeling of the path $P(T)$

Let $v_{i} v_{j}$ be any edge of $T$ with $1 \leq i<j \leq n$ and $P_{1}$ be the ept that deletes this edge and add the edge $v_{i+t} v_{j-t}$ where $t$ is the distance of $v_{i}$ from $v_{i+t}$ and also the distance of $v_{j}$ from $v_{j-t}$. Let $P$ be a parallel transformation of $T$ that contains $P_{1}$ as one of the constituent epts.

Since $v_{i+t} v_{j-t}$ is an edge of the path $P(T)$, it follows that $i+t+1=j-t$ which implies $j=i+2 t+1$. Therefore $i$ and $j$ are of opposite parity.

The induced label of the edge $v_{i} v_{j}$ is given by

$$
\begin{aligned}
f^{*}\left(v_{i} v_{j}\right) & =f^{*}\left(v_{i} v_{i+2 t+1}\right) \\
& =f\left(v_{i}\right)+f\left(v_{i+2 t+1}\right) \\
& = \begin{cases}\frac{i}{2}+\frac{i+2 t}{2} & \text { if } i \text { is even } \\
\frac{i-1}{2}+\frac{i+2 t+1}{2} & \text { if } i \text { is odd }\end{cases} \\
& =i+t, 1 \leq i \leq n
\end{aligned}
$$

Now

$$
\begin{aligned}
f^{*}\left(v_{i+t} v_{j-t}\right) & =f^{*}\left(v_{i+t} v_{i+t+1}\right) \\
& =f\left(v_{i+t}\right)+f\left(v_{i+t+1}\right) \\
& =i+t, 1 \leq i \leq n .
\end{aligned}
$$

Therefore, we have $f^{*}\left(v_{i} v_{j}\right)=f^{*}\left(v_{i+t} v_{j-t}\right)$ and hence $f$ is a vertex equitable labeling of the $T_{p}$-tree $T$.

An example for the vertex equitable labeling of a $T_{p^{-}}$ tree with 12 vertices is given in Figure 2.

Theorem 2.4 Let $T$ be a $T_{p}$-tee with even number of vertices. Then the graph $T \odot \bar{K}_{n}$ is a vertex equitable graph for all $n \geq 1$.

Proof. Let $T$ be a $T_{p}$-tree of even order $m$ and the vertex set $V(T)=\left\{v_{1}, v_{2}, v_{3}, \cdots, v_{m}\right\}$. Let $u_{1}^{j}, u_{2}^{j}, \cdots, u_{n}^{j}$ be the pendant vertices joined with $v_{j}(1 \leq j \leq m)$ by an edge. Then

$$
V\left(T \odot \overline{K_{n}}\right)=\left\{v_{j}, u_{i}^{j}: 1 \leq i \leq n, 1 \leq j \leq m\right\} .
$$

By the definition of a $T_{p}$-tree, there exists a parallel transformation $P$ of $T$ such that for the path $P(T)$ we have 1) $V(P(T))=V(T)$,2) $E(P(T))=\left(E(T)-E_{d}\right) \cup E_{p}$ where $E_{d}$ is the set of edges deleted from $T$ and $E_{p}$ is the set of edges newly added through the sequence $P=\left(P_{1}, P_{2}, \cdots, P_{k}\right)$ of the epts $P$ used to arrive the path $P(T)$. Clearly, $E_{d}$ and $E_{p}$ have the same number of edges.

Now denote the vertices of $P(T)$ successively as $v_{1}, v_{2}, \cdots, v_{m}$ starting from one pendant vertex of $P(T)$ right up to the other. The labeling $f$ defined by

$$
f\left(v_{j}\right)= \begin{cases}\frac{(n+1)(j-1)}{2} & \text { if } j \text { is odd } \\ \frac{(n+1) j}{2} & \text { if } j \text { is even, }\end{cases}
$$

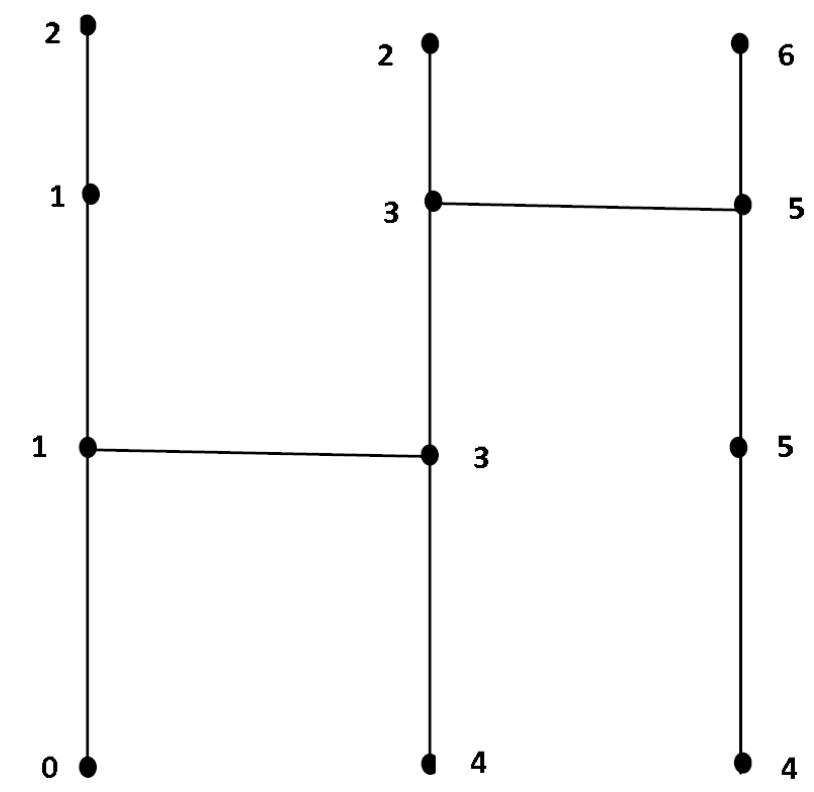

Figure 2. Vertex equitable labeling of a $T_{p}$-tree with 12 vertices. 


$$
f\left(u_{i}^{j}\right)=\left\{\begin{array}{ll}
\frac{(n+1)(j-1)}{2}+i & \text { if } j \text { is odd } \\
\frac{(n+1)(j-2)}{2}+i & \text { if } j \text { is even. }
\end{array}, 1 \leq i \leq n\right.
$$

is a vertex equitable labeling graph.

Let $v_{i} v_{j}$ be any edge of $T$ with $1 \leq i<j \leq m$ let $P_{1}$ be the ept that deletes this edge and adds the edge $v_{i+t} v_{j-t}$ where $t$ is the distance of $v_{i}$ from $v_{i+t}$ and also the distance of $v_{j}$ from $v_{j-t}$. Let $P$ be a parallel transformation of $T$ that contains $P_{1}$ as one of the constituent epts.

Since $v_{i+t} v_{j-t}$ is an edge in the path $P(T)$, it follows that $i+t+1=j-t$ which implies $j=i+2 t+1$. Therefore $i$ and $j$ are of opposite parity.

The induced label of the edge $v_{i} v_{j}$ is given by

$$
\begin{aligned}
f^{*}\left(v_{i} v_{j}\right) & =f^{*}\left(v_{i} v_{i+2 t+1}\right)=f\left(v_{i}\right)+f\left(v_{i+2 t+1}\right) \\
& = \begin{cases}\frac{(n+1) i}{2}+\frac{(n+1)(i+2 t+1-1)}{2} & \text { if } i \text { is even } \\
\frac{(n+1)(i-1)}{2}+\frac{(n+1)(i+2 t+1)}{2} & \text { if } i \text { is odd }\end{cases} \\
& =(n+1)(i+t), 1 \leq i \leq n \\
f^{*}\left(v_{i+t} v_{j-t}\right) & =f^{*}\left(v_{i+t} v_{i+t+1}\right)=f\left(v_{i+t}\right)+f\left(v_{i+t+1}\right) \\
& =(n+1)(i+t), 1 \leq i \leq n .
\end{aligned}
$$

Therefore, we have $f^{*}\left(v_{i} v_{j}\right)=f\left(v_{i+t} v_{j-t}\right)$ and thus $f$ is a vertex equitable labeling of $T \odot \overline{K_{n}}$.

An example for the vertex equitable labeling of $T \odot \overline{K_{5}}$, where $T$ is a $T_{p}$-tree with 12 vertices is shown in Figure 3.

Let $B(n, n+1)$ be a graph obtained from $K_{2}$ by attaching $n$ pendant edges at one vertex and $n+1$ pendant edges at the other vertex.

Theorem 2.5 The bistar $B(n, n+1)$ is a vertex equitable graph.

Proof. Let $V\left(K_{2}\right)=\{u, v\}$ and $u_{i}(1 \leq i \leq n)$ and $v_{j}(1 \leq j \leq n+1)$ be the vertices adjacent to $u$ and $v$ respectively. Now, $B(n, n+1)$ has $2 n+2$ edges and $2 n+3$ vertices. Define

$$
f: V(B(n, n+1)) \rightarrow\left\{0,1,2, \cdots,\left\lceil\frac{2 n+2}{2}\right\rceil\right\}
$$

by $f(u)=0, f(v)=n+1, f\left(u_{i}\right)=i$ if $1 \leq i \leq n$ and $f\left(v_{j}\right)=j$ if $1 \leq j \leq n+1$. Then $f$ is a vertex equitable labeling of $B(n, n+1)$.

Theorem 2.6 Let $x_{1}<x_{2} \leq x_{3} \leq \cdots \leq x_{n}$ and

$$
k=\left\{\begin{array}{r}
\left(x_{2}+x_{4}+\cdots+x_{n}\right)-\left(x_{1}+x_{3}+\cdots+x_{n-1}\right)-1 \\
\text { if } n \text { is even } \\
\left(x_{1}+x_{3}+\cdots+x_{n}\right)-\left(x_{2}+x_{4}+\cdots+x_{n-1}\right) \\
\text { if } n \text { is odd }
\end{array}\right.
$$

Then $S\left(x_{1}, x_{2}, \cdots, x_{n}, k+1\right)$ is a vertex equitable graph.

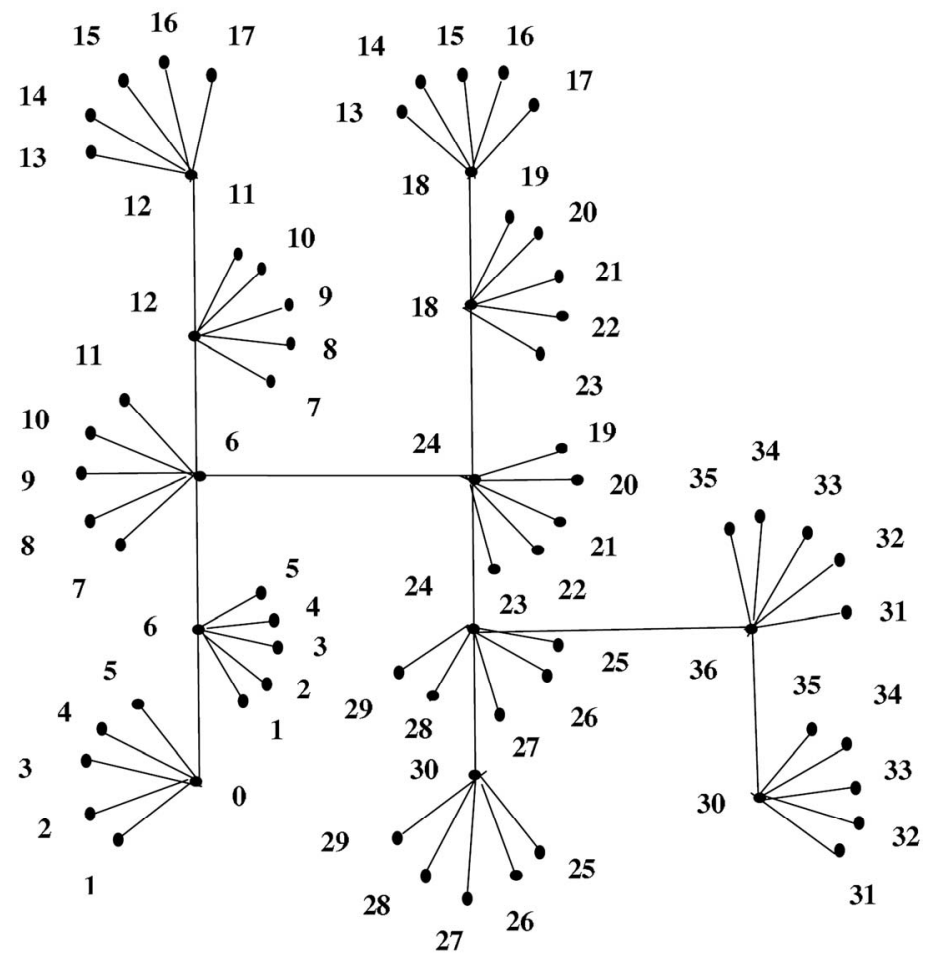

Figure 3. Vertex equitable labeling of $T \odot \overline{K_{5}}$. 
Proof. By Theorem 2.5, $S\left(x_{1}, x_{1}+1\right)$ is a vertex equitable graph. Let $f_{1}$ be the corresponding vertex equitable labeling of $S\left(x_{1}, x_{1}+1\right)$. Let $y_{1}=x_{2}-\left(x_{1}+1\right)$. Since $x_{1}<x_{2}, y_{1} \geq 0$. Consider the graphs $S\left(x_{1}, x_{1}+1\right)$ and $S\left(y_{1}, y_{1}+1\right)$. The number of edges of the graph $S\left(x_{1}, x_{1}+1\right)$ is $2 x_{1}+2$.

Now,

$$
\left(S\left(x_{1}, x_{1}+1\right)\right)_{f_{1}} *\left(S\left(y_{1}, y_{1}+1\right)\right)_{f_{1}}
$$

$$
=S\left(x_{1}, x_{1}+y_{1}+1, y_{1}+1\right)=S\left(x_{1}, x_{2}, y_{1}+1\right) \text {. }
$$

Therefore, by Theorem 2.1, $S\left(x_{1}, x_{1}+y_{1}+1, y_{1}+1\right)$ is a vertex equitable graph. Let $f_{2}$ be the corresponding vertex equitable labeling of $S\left(x_{1}, x_{2}, y_{1}+1\right)$. Again the number of edges of $S\left(x_{1}, x_{2}, y_{1}+1\right)$ is even.

Now take $y_{2}=x_{3}-\left(y_{1}+1\right)=x_{3}-x_{2}+x_{1}+1-1$. Hence $y_{2} \geq 0$. Also

$$
\begin{aligned}
& \left(S\left(x_{1}, x_{2}, y_{1}+1\right)\right)_{f_{2}} *\left(S\left(y_{2}, y_{2}+1\right)\right)_{f_{1}} \\
= & S\left(x_{1}, x_{2}, y_{1}+y_{2}+1, y_{2}+1\right) \\
= & S\left(x_{1}, x_{2}, x_{3}, y_{2}+1\right) .
\end{aligned}
$$

Therefore, by Theorem 2.1, $S\left(x_{1}, x_{2}, x_{3}, y_{2}+1\right)$ is a vertex equitable graph and the number of edges is even. Proceeding like this, at the $(n-2)^{t h}$ step we get $S\left(x_{1}, x_{2}, x_{3}, \cdots, x_{n-1}, y_{n-2}+1\right)$ is a vertex equitable graph where

$$
y_{n-2}=\left\{\begin{array}{r}
\left(x_{2}+x_{4}+\cdots+x_{n-1}\right)-\left(x_{1}+x_{3}+\cdots+x_{n-2}\right)-1 \\
\text { if } n \text { is even } \\
\left(x_{1}+x_{3}+\cdots+x_{n-1}\right)-\left(x_{2}+x_{4}+\cdots+x_{n-2}\right) \\
\text { if } n \text { is odd }
\end{array}\right.
$$

Let $f_{n-1}$ be the corresponding vertex equitable labeling of $S\left(x_{1}, x_{2}, x_{3}, \cdots, x_{n-1}, y_{n-2}+1\right)$. Take

$$
y_{n-1}=\left\{\begin{array}{r}
\left(x_{2}+x_{4}+\cdots+x_{n}\right)-\left(x_{1}+x_{3}+\cdots+x_{n-1}\right)-1 \\
\text { if } n \text { is even } \\
\left(x_{1}+x_{3}+\cdots+x_{n}\right)-\left(x_{2}+x_{4}+\cdots+x_{n-1}\right) \\
\text { if } n \text { is odd }
\end{array}\right.
$$

Clearly $y_{n-2}+y_{n-1}+1=x_{n}$. Now,

$$
\begin{aligned}
& \left(S\left(x_{1}, x_{2}, x_{3}, \cdots, x_{n-1}, y_{n-2}+1\right)\right)_{f_{n-1}} *\left(S\left(y_{n-1}, y_{n-1}+1\right)\right)_{f_{1}} \\
= & S\left(x_{1}, x_{2}, x_{3}, \cdots, x_{n-1}, y_{n-2}+1+y_{n-1}, y_{n-1}+1\right) \\
= & S\left(x_{1}, x_{2}, x_{3}, \cdots, x_{n}, k+1\right) .
\end{aligned}
$$

Therefore, $S\left(x_{1}, x_{2}, x_{3}, \ldots, x_{n-1}, x_{n}, k+1\right)$ is a vertex equitable graph.

An example for the vertex equitable labeling of $S(4,6,9,7+1)$ if $n$ is odd is given in Figure 4 .

An example for the vertex equitable labeling of $S(5,7,9,10,2+1)$ if $n$ is even is given in Figure 5.

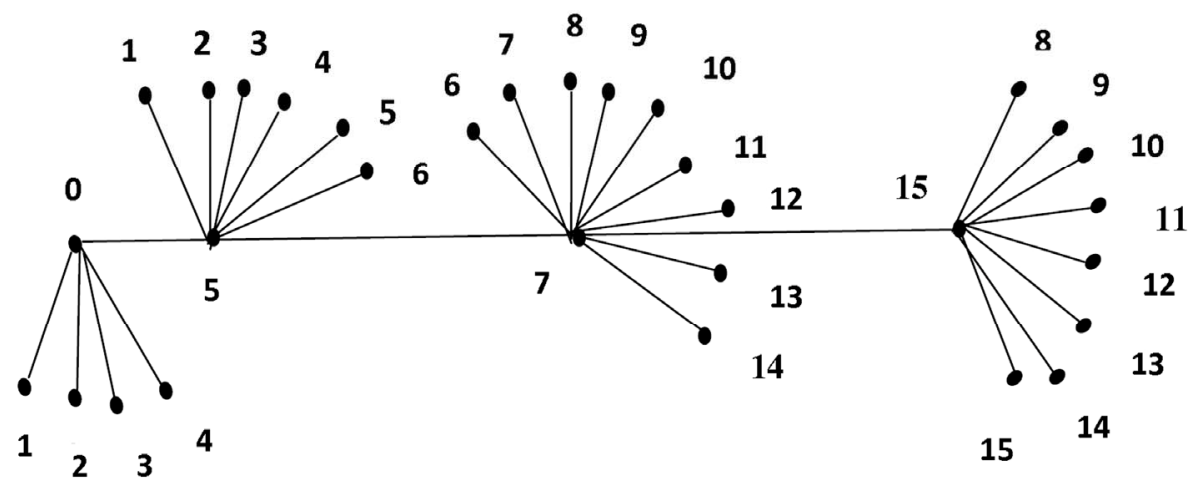

Figure 4. Vertex equitable labeling of $S(4,6,9,7+1)$.

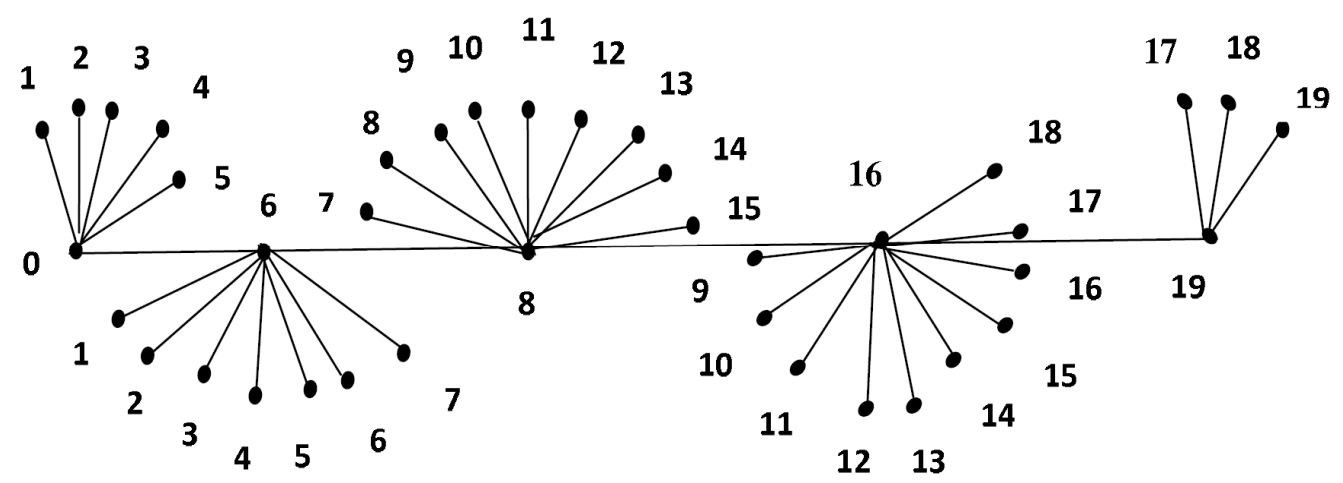

Figure 5. Vertex equitable labeling of $S(5,7,9,10,2+1)$. 
Theorem 2.7 The crown $C_{n} \odot K_{1}$ is a vertex equitable graph.

Proof: Let $u_{1}, u_{2}, \cdots u_{n}$ be the vertices of the cycle $C_{n}$ and let $v_{i}$ be the vertex adjacent to $u_{i}$ for $1 \leq i \leq n$. Then the vertex set $V\left(C_{n} \odot K_{1}\right)=\left\{u_{i}, v_{i}: 1 \leq i \leq n\right\}$ and the edge set $E\left(C_{n} \odot K_{1}\right)=\left\{u_{i} u_{i+1}, u_{i} v_{i}, u_{n} u_{1} u_{n} v_{n},: 1 \leq i \leq n-1\right\}$. Define $f: V\left(C_{n} \odot K_{1}\right) \rightarrow\{0,1,2 \cdots, n\}$ for the following cases:

Case 1. $n \equiv 0(\bmod 4)$.

$$
\begin{aligned}
& f\left(u_{i}\right)= \begin{cases}(i-1) & \text { for } i=1,3,5, \cdots, \frac{n}{2}-1 \\
i & \text { for } i=2,4,6, \cdots, \frac{n}{2} \\
i & \text { for } \frac{n}{2}+1 \leq i \leq n,\end{cases} \\
& f\left(v_{i}\right)= \begin{cases}i & \text { for } i=1,3,5, \cdots, \frac{n}{2}-1 \\
(i-1) & \text { for } i=2,4,6, \cdots, \frac{n}{2} \\
i & \text { for } \frac{n}{2}+1 \leq i \leq n .\end{cases}
\end{aligned}
$$

Case 2. $n \equiv 1(\bmod 4)$.

$$
\begin{aligned}
& f\left(u_{i}\right)= \begin{cases}i & \text { for } i=1,3,5, \cdots,\left\lceil\frac{n}{2}\right\rceil \\
i-1 & \text { for } i=2,4,6, \cdots,\left\lfloor\frac{n}{2}\right\rceil \\
i & \text { for }\left\lceil\frac{n}{2}\right\rceil+1 \leq i \leq n,\end{cases} \\
& f\left(v_{i}\right)= \begin{cases}i-1 & \text { for } i=1,3,5, \cdots,\left\lceil\frac{n}{2}\right\rceil \\
i & \text { for } i=2,4,6, \cdots,\left\lfloor\frac{n}{2}\right\rfloor \\
i & \text { for }\left\lceil\frac{n}{2}\right\rceil+1 \leq i \leq n .\end{cases}
\end{aligned}
$$

Case 3. $n \equiv 2(\bmod 4)$.

$$
f\left(u_{i}\right)= \begin{cases}(i-1) & \text { for } i=1,3,5, \cdots, \frac{n}{2} \\ i & \text { for } i=2,4,6, \cdots, \frac{n}{2}-1 \\ i & \text { for } \frac{n}{2}+2 \leq i \leq n,\end{cases}
$$

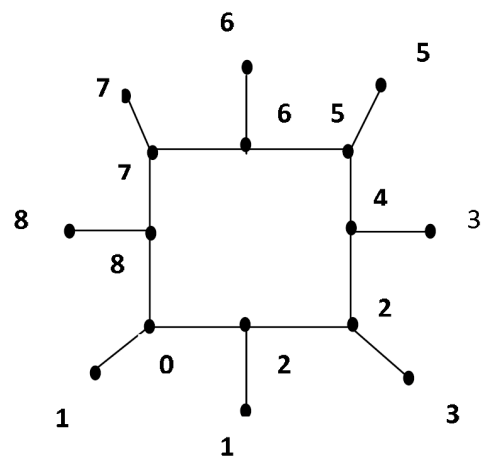

Figure 6. Vertex equitable labeling of $C_{8} \odot K_{1}$.

$$
\begin{aligned}
& f\left(v_{i}\right)= \begin{cases}i & \text { for } i=1,3,5, \cdots, \frac{n}{2} \\
(i-1) & \text { for } i=2,4,6, \cdots, \frac{n}{2}-1 \\
i & \text { for } \frac{n}{2}+3 \leq i \leq n .\end{cases} \\
& f\left(v_{(n / 2)+1}\right)=\frac{n}{2}, \quad f\left(v_{(n / 2)+2}\right)=\frac{n}{2}+1, \\
& f\left(u_{(n / 2)+1}\right)=\frac{n}{2}+2 .
\end{aligned}
$$

Case 4. $n \equiv 3(\bmod 4)$.

$$
\begin{aligned}
& f\left(u_{i}\right)= \begin{cases}(i-1) & \text { for } i=1,3,5, \cdots,\left\lfloor\frac{n}{2}\right\rfloor \\
i & \text { for } i=2,4,6, \cdots,\left\lceil\frac{n}{2}\right\rceil \\
i & \text { for }\left\lceil\frac{n}{2}\right\rceil+1 \leq i \leq n,\end{cases} \\
& f\left(v_{i}\right)= \begin{cases}i \quad \text { for } i=1,3,5, \cdots,\left\lfloor\frac{n}{2}\right\rfloor \\
(i-1) & \text { for } i=2,4,6, \cdots,\left\lceil\frac{n}{2}\right\rceil-1 \\
i & \text { for }\left\lceil\frac{n}{2}\right\rceil \leq i \leq n .\end{cases}
\end{aligned}
$$

In all the above cases, $f$ is a vertex equitable labeling. Hence $C_{n} \odot K_{1}$ is a vertex equitable graph.

An example for the vertex equitable labeling of $C_{8} \odot K_{1}$ is shown in Figure 6.

Theorem 2.8 The graph $P_{n}^{2}$ is a vertex equitable graph.

Proof. Let $u_{1}, u_{2}, \cdots, u_{n}$ be the path $P_{n}$. Clearly, $P_{n}^{2}$ has $n$ vertices and $2 n-3$ edges. Define

$$
f: V\left(P_{n}^{2}\right) \rightarrow\left\{0,1,2, \cdots,\left\lceil\frac{2 n-3}{2}\right\rceil\right\}
$$


by $f\left(u_{i}\right)=i-1,1 \leq i \leq n$. Evidently, $P_{n}^{2}$ is a vertex equitable graph.

\section{REFERENCES}

[1] F. Harary, "Graph Theory," Addison Wesley, Massachusetts, 1972.

[2] J. A. Gallian, "A Dynamic Survey of Graph Labeling,” The Electronic Journal of Combinatorics, Vol. 18, 2011, Paper \#DS6.

[3] G. Bloom and S. Ruiz, "Decomposition into Linear Forest and Difference Labelings of Graphs,” Discrete Applied Mathematics, Vol. 49, 1994, pp. 61-75. doi:10.1016/0166-218X(94)90201-1

[4] S. M. Hegde and S. Shetty, "On Graceful Trees," Applied Mathematics E-Notes, Vol. 2, 2002, pp. 192-197.

[5] R. Ponraj and S. Somasundram, "Mean Labeling of Graphs,” National Academy Science Letters, Vol. 26, 2003, pp. 210-213.
[6] R. Ponraj and S. Somasundram, "Non-Existence of Mean Labeling for a Wheel," Bulletin of Pure and Applied Sciences (Mathematics \& Statistics), Vol. 22E, 2003, pp. 103-111.

[7] R. Ponraj and S. Somasundram, "Some Results on Mean Graphs,” Pure and Applied Mathematical Sciences, Vol. 9, 2004, pp. 47-58.

[8] R. Ponraj and S. Somasundram, "Further Results on Mean Graphs," Proceedings f SACOEFERENCE, National Level Conference, Dr. Sivanthi Aditanar College of Engineering, 2005, pp. 443-448.

[9] M. Seenivasan and A. Lourdusamy, "Vertex Equitable Labeling of Graphs," Journal of Discrete Mathematical Sciences \& Cryptography, Vol. 11, No. 6, 2008, pp. 727 735.

[10] P. Jeyanthi and A. Maheswari, “On Vertex equitable labeling," Preprint.

[11] P. Jeyanthi and A. Maheswari, "Vertex Equitable Labeling of Cycle and Path Related Graphs,” Utilitas Mathematica, Article in Press. 